\title{
Auditory and Motor Contributions to the Timing of Melodies Under Cognitive Load
}

\author{
Pieter-Jan Maes \\ Ghent University
}

\author{
Madison Giacofci \\ Leuven University
}

\author{
Marc Leman \\ Ghent University
}

\begin{abstract}
Current theoretical models and empirical research suggest that sensorimotor control and feedback processes may guide time perception and production. In the current study, we investigated the role of motor control and auditory feedback in an interval-production task performed under heightened cognitive load. We hypothesized that general associative learning mechanisms enable the calibration of time against patterns of dynamic change in motor control processes and auditory feedback information. In Experiment 1, we applied a dual-task interference paradigm consisting of a finger-tapping (continuation) task in combination with a working memory task. Participants (nonmusicians) had to either perform or avoid arm movements between successive key presses (continuous vs. discrete). Auditory feedback from a key press (a piano tone) filled either the complete duration of the target interval or only a small part (long vs. short). Results suggested that both continuous movement control and long piano feedback tones contributed to regular timing production. In Experiment 2, we gradually adjusted the duration of the long auditory feedback tones throughout the duration of a trial. The results showed that a gradual shortening of tones throughout time increased the rate at which participants performed tone onsets. Overall, our findings suggest that the human perceptual-motor system may be important in guiding temporal behavior under cognitive load.
\end{abstract}

Keywords: interval production, associative learning, internal model, sensorimotor adaptation

For decades, the main challenge of research on time perception and production was to identify the locus of a hypothesized internal clock inside our heads (Allman, Teki, Griffiths, \& Meck, 2014; Church, 1984). Although it is now commonly agreed that there is no such single, dedicated locus in the human brain, it is assumed that some sort of general-purpose, cognitively controlled internal clock mechanism is available for keeping track of time (Allman et al., 2014). This timekeeper hypothesis is supported by research showing that an additional cognitive task interferes with the production of regularly timed intervals (Brown, 1997; Fortin \& Breton, 1995; Krampe, Doumas, Lavrysen, \& Rapp, 2010; Ogden,

This article was published Online First June 22, 2015.

Pieter-Jan Maes, Institute for Psychoacoustics and Electronic Music, Department of Art, Music and Theatre Sciences, Ghent University; Madison Giacofci, Statistics Section, Department of Mathematics, Leuven University; Marc Leman, Institute for Psychoacoustics and Electronic Music, Department of Art, Music and Theatre Sciences, Ghent University.

This research was conducted in the framework of the EmcoMetecca project, granted by the Ghent University-Methusalem-BOF Council. We want to thank Sara Colin and Sven Sabbe for contributions to the data acquisition part of the study and Ivan Schepers for technical assistance.

Correspondence concerning this article should be addressed to PieterJan Maes, Institute for Psychoacoustics and Electronic Music, Department of Art, Music and Theatre Sciences, Ghent University, SintPietersnieuwstraat 41,9000 Ghent, Belgium. E-mail: pieterjan.maes@ UGent.be
Salominaite, Jones, Fisk, \& Montgomery, 2011; Rattat, 2010). However, because the computational and cognitive resources of humans are limited, and temporal production often occurs in situations of cognitive load, the timekeeper hypothesis is presumably inadequate to fully explain timing behavior. At the end of the 20th century, ecological and embodied theories shifted focus from abstract, internal control processes within the brain to sensorimotor interactions with the external environment to explain cognition and behavior, suggesting a new perspective on timing rooted in embodiment (Gibson, 1979; Lakoff \& Johnson, 1999; Varela, Rosch, \& Thompson, 1991). Current research suggests that the perceptual and motor systems, and their coupling, can support time perception and timing production (Hopson, 2003; M. R. Jones \& Boltz, 1989; Mauk \& Buonomano, 2004; Ross \& Balasubramaniam, 2014).

Research on motor control and coordination suggests that the timing of rhythmic body movements is a hybrid phenomenon, in the sense that different control systems are recruited depending on movement type and production rate. Concerning movement type, a distinction is made between discrete and continuous rhythmic (quasiperiodic) movements (Braun Janzen, Thompson, Ammirante, \& Ranvaud, 2014; Delignières, Lemoine, \& Torre, 2004; LaRue, 2005; Robertson et al., 1999; Studenka, Zelaznik, \& Balasubramaniam, 2012; Torre \& Balasubramaniam, 2009; Zelaznik, Spencer, \& Ivry, 2002, 2008). Whereas discrete rhythmic movements are characterized by salient events separated by pauses, continuous rhythmic movements are smooth, without interspersed pauses. Importantly, research suggests that these move- 
ment types rely on different control mechanisms. Discrete movements are regulated by an event-based timing system, which closely resembles the timekeeper hypothesis. In contrast, continuous movements are regulated by an emergent timing system, which pertains to a dynamical systems perspective on motor control. According to this perspective, coordinated (regular) body movements are to a high extent the result of the motor system's dynamics, with a minimum of explicit, central control (Kelso, 1997; Thelen, 1991; Turvey, 1977; Warren, 2006). Concerning production rate, research has indicated that the mechanisms underlying the temporal control of rhythmic movements depend on the duration of the intervals (i.e., suprasecond or subsecond) that need to be timed (Lewis \& Miall, 2003). The production of brief, subsecond intervals - interonset intervals (IOIs) $<1 \mathrm{~s}$-is thought to be regulated by an automatic system that capitalizes on motor system functions (e.g., supplementary motor area, left sensorimotor cortex). In contrast, the production of longer, suprasecond intervals (IOIs $>1 \mathrm{~s}$ ) is cognitively controlled depending on prefrontal and parietal regions.

Music performance provides an excellent context in which to study how people may capitalize on their sensorimotor capabilities in interaction with their environment to regulate timing behavior. Moreover, the use of timing strategies that capitalize on sensorimotor processes is especially relevant in this context, because musicians, in particular novice musicians, often perform under heightened cognitive load. A recent study investigated the effects of heightened cognitive load on the production of regular intervals in the context of cello performance (Maes, Wanderley, \& Palmer, 2015). In an attempt to provide empirical support for the theories related to production rate and movement type discussed earlier, intervals were either subsecond or suprasecond and needed to be produced by means of either discrete or continuous movements. In agreement with the theories, the results indicated that only slow production rates (i.e., suprasecond intervals) produced by discrete rhythmic movements were disturbed by an additional working memory task (a digit-switch task). This was reflected in a significant increase in the overall variability of produced interval durations. The finding that continuous rhythmic movements are relatively unharmed by additional cognitive load suggests that temporal control may rely, at least in part, on other than cognitive resources shared with working memory task performance-presumably resources that relate to sensorimotor processing. However, the main limitation of the study was that no differentiation could be made between the relative contributions of the motor system and the auditory feedback that resulted from performed movements; bow strokes (legato vs. staccato) were coupled to the produced tones (long vs. short). Previous research has indicated that self-generated auditory feedback may support and influence regular timing production. This seems especially to be the case in the learning of new motor skills. For instance, van Vugt and Tillmann (2015) demonstrated that when people were asked to tap regularly, overall tapping regularity improved when people perceived sounds synchronously with their keystrokes. Also, Repp (1999) showed that the removal of auditory feedback during keyboard performances resulted in significant (although small) effects on all expressive parameters, in particular peddling. In addition, studies have indicated that changes in acoustics, such as reverberation time, may have a substantial impact on the timing perfor- mance of pianists (Bolzinger \& Risset, 1992; Furuya \& Soechting, 2010).

In this article, we present a study that followed up on the finding of Maes et al. (2015) that temporal regularity of continuous rhythmic movements at slow tempi is relatively unaffected by a heightened cognitive load compared with temporal regularity of discrete rhythmic movements. More particularly, we aim to deliver more insights into the relative roles of motor production and auditory feedback in support of temporal regularity under cognitive load. For that purpose, we created a design in which we manipulated movement type (discrete, continuous) and auditory feedback (short, long) in a typical synchronization-continuation tapping task within a dual-task paradigm. For this study, we focused on nonmusicians for two reasons. First, research has shown that sensory feedback is most beneficial in the learning stages of motor skill acquisition (Adams, 1971; Bishop, Bailes, \& Dean, 2014; Finney \& Palmer, 2003; Takahashi \& Tsuzaki, 2008; van Vugt \& Tillmann, 2015). Second, musical novices who want to learn to play a musical instrument are expected to benefit most from a timing strategy based on sensorimotor mechanisms, because they often perform under heightened cognitive load. Also, we focused on the production of suprasecond intervals because only in that case were continuous movements shown to benefit regular interval production (Maes et al., 2015).

In addition, we want to put forward and elaborate on some important theoretical concepts that could broaden understanding of how sensorimotor interaction processes may regulate and guide temporal behavior. In particular, we bring up two mechanisms that relate to the concept of internal models, which is a crucial concept in the study of motor control and sensory processing (Kawato, 1999; Maes, Leman, Palmer, \& Wanderley, 2014; Wolpert, Ghahramani, \& Jordan, 1995). The first mechanism is a general (associative) learning mechanism that enables an individual to calibrate time against patterns of dynamic change in motor control and sensory feedback information. The second mechanism, auditorymotor adaptation, relies on the first. When a temporal misalignment occurs in a previously established relationship between motor control and sensory feedback information (i.e., a prediction error), people will automatically adapt their behavior to reduce this misalignment as much as possible. In the following two sections, we discuss these mechanisms in more detail.

\section{General Associative Learning Mechanisms}

Our approach to timing production emphasizes the role of the dynamic interaction between an agent, with its sensorimotor capabilities, and the external environment. To understand this approach better, we refer to the concept of an hourglass (in its actual use, not as metaphor as in the scalar expectancy theory; Gibbon, Church, \& Meck, 1984; Ivry \& Richardson, 2002). Basically, an hourglass is an object that is used to measure a temporal interval by a sand flow going from a top bulb, through a small neck, to a bottom bulb. Once all the sand has run to the bottom bulb, the hourglass can be inverted and the process repeated. If everything is kept unchanged, inverting the hourglass each time that all the sand has passed from the top bulb to the bottom bulb will demarcate a constant, regular interval. This strategy enables measurement of a temporal interval without explicit computation of the passage of time. Rather, the passage of time is physically encoded 
in the sand flow from the one bulb to the other, and regular interval production may be realized through mere sensorimotor interaction (i.e., perceiving the flow of sand and inverting the hourglass). Besides a sand flow going from one bulb to another, there are plenty of other physical phenomena that may unfold over time in a regular way, with sound and body movement being two particularly relevant examples. For instance, striking a piano key produces a tone the amplitude of which gradually decays over time, depending specifically on type of piano, striking velocity, and room acoustics. Regular timing may then be realized by anchoring actions (e.g., striking of keys) to patterns of dynamic change inherent to the sensory information (e.g., amplitude decay; Bravi et al., 2014; Rodger \& Craig, 2011; Roerdink, Ridderikhoff, Peper, \& Beek, 2013; Varlet, Marin, Issartel, Schmidt, \& Bardy, 2012). For instance, repeatedly and similarly striking a piano key just after the preceding tone has ceased results in a regular auditory pattern. In addition, a pianist can perform particular movements of the arms, head, and other body parts between successive keystrokes. Compared with an hourglass, auditory-motor patterns are more dynamic, because they can be entrained to an external rhythmic auditory stimulation, such as a metronome or music (cf. dance, music performance, and sports practice).

Through repeated experience, associations may be established between sensorimotor regularities in relation to the passage of time. Accordingly, time may become calibrated against auditorymotor patterns, which may later on function like an hourglass to keep track of the passage of time and guide temporal behavior (Addyman, French, Mareschal, \& Thomas, 2011; Hopson, 2003). The temporal integration of actions and their sensory outcome establishes what is typically referred to as an internal model (Maes et al., 2014). Internal models are acquired during development, as a result of systematically repeated sensorimotor experiences. One of the dominant theories explaining the underlying mechanisms of this process is Heyes and Ray's (2000) theory of associative sequence learning.

\section{Auditory-Motor Adaptation}

Internal models that capture relationships between actions and their sensory outcomes contain an inverse component and a forward component. Inverse models allow inference from sensory representations of the corresponding motor commands required to generate a specific sensory state. In contrast, forward models allow prediction of the likely sensory outcome of a planned or executed action. A distinct property of forward models is that they allow transformation of discrepancies between the expected and the actual sensory outcome of a performed action into an error signal, which drives changes in motor output to reduce sensory prediction errors (Friston, Kilner, \& Harrison, 2006; Jordan \& Rumelhart, 1992; Lalazar \& Vaadia, 2008; Shadmehr, Smith, \& Krakauer, 2010; van der Steen \& Keller, 2013; Wolpert et al., 1995).

Referring back to our hourglass example, changing one or more of the characteristics of the hourglass (e.g., amount of sand, neck width, sand quality) will have an impact on the interval duration that is measured. For instance, suppose the neck width of the hourglass gets gradually thinner; successive intervals demarcated by the hourglass will be lengthened accordingly. Hence, when a merely perceptual-motor strategy is used-that is, waiting until all sand has run to the bottom bulb before inverting the hourglass- temporal behavior will be affected by changes in the hourglass parameters to keep the relationship between perception and action consistent over time.

Motor adaptation is exactly that process in which motor commands are updated in response to altered environmental conditions. Sensorimotor adaptation is typically studied in the context of sensorimotor synchronization to external sensory stimuli (Elliott, Wing, \& Welchman, 2014; Loehr, Large, \& Palmer, 2011; Palmer, Lidji, \& Peretz, 2014; Repp \& Su, 2013; van der Steen \& Keller, 2013). Research has identified two (independent) error-correction processes that occur in response to either period or phase perturbations and that enable people to stay in synchrony with an external stimulus. In line with the scope of our study, we are more interested in motor adaptation in response to unexpected alterations of self-generated sensory (here, auditory) feedback. This form of motor adaptation is typically studied by using error-based, or perturbation, paradigms, which alter the sensory outcome of well-learned movements. In this field of research, most attention has been devoted to visual and vestibular perturbation paradigms using prisms, rotations, force fields, and so forth to alter expected visual and vestibular feedback of performed actions (for reviews, see Krakauer \& Mazzoni, 2011; Welch, 1986). In addition, a considerable body of research has been conducted on auditorymotor adaptation in the domain of speech production. One of the first such studies was conducted by Houde and Jordan (1998). Participants were prompted to produce consonant-vowelconsonant words. Over many trials, the real-time auditory feedback heard by participants was increasingly altered by shifting the three lowest formant frequencies and, hence, changing the vowel's perceived phonetic identity. Results showed that the speech motorcontrol system responded adaptively to alterations of sensory feedback by adjusting the produced formant frequencies in the opposite direction of the alteration. In a recent study by Keough, Hawco, and Jones (2013), participants were asked to match the pitch of their voice to a musical target. They were informed that their auditory feedback would be altered in pitch, and they were asked to either compensate for or ignore any alterations. In both cases, it was shown that participants were not able to suppress compensatory responses and auditory-motor adaptation (i.e., they adjusted their voice fundamental frequency in the opposite direction of the alteration). Other relevant studies in this context include J. A. Jones and Munhall (2000); Villacorta, Perkell, and Guenther (2007); Feng, Gracco, and Max (2011); Hickok, Houde, and Rong (2011); Mollaei, Shiller, and Gracco (2013); and Shiller and Rochon (2014). In the context of music performance, auditory feedback is naturally influenced by room acoustics. Previous research has demonstrated that playing the piano in rooms with different reverberation times has an impact on performed tempos-more specifically, the longer the reverberation time, the slower one tends to play, and vice versa (Bolzinger \& Risset, 1992; Kawai, Harada, Kato, Ueno, \& Sakuma, 2013). This finding suggests that, similar to the hourglass example, people may spontaneously adapt to changing environmental conditions to keep the (expected) relationship between action and perception consistent over time.

In summary, the main aim of the present experiments was to follow up on the finding of Maes et al. (2015) that the production of suprasecond regular temporal intervals by means of continuous movements is significantly less disturbed by an additional cogni- 
tive load task compared with discrete movements. In the current study, we sought to provide more detailed knowledge about the relative contributions of motor performance and auditory feedback to this effect. We designed two experiments to test the theoretical hypotheses we have put forward-with associative learning and motor adaptation as central concepts-to explain in more detail how sensorimotor interaction may provide a strategy to regulate temporal behavior.

\section{Experiment 1}

Experiment 1 involved a typical synchronization-continuation tapping task in which people were asked to synchronize piano tones to an auditory metronome by tapping a key (synchronization phase) and to keep that regular pace for a certain extent of time after the metronome has stopped (continuation phase; Stevens, 1886; Wing \& Kristofferson, 1973). This task was performed in combination with an additional working memory task (digit-switch task) and compared with a baseline condition in which the tapping task was performed without the working memory task. The tapping task was performed under different conditions related to the movement type people used to tap and the auditory feedback they received when tapping the key. People were asked either to perform continuous arm movements between successive key presses (continuous movement type) or to restrain from making body movements between key presses (discrete movement type). In addition, tapping a key generated either a short piano tone (short auditory feedback) or a long piano tone filling the complete duration of the interval to be timed (long auditory feedback). These conditions were fully crossed to yield four different tapping conditions. Following up on the study by Maes et al. (2015), the aim of the experiment was to assess the relative contributions of movement type (continuous, discrete) and auditory feedback (short, long) to regular interval production under cognitive load. In the tapping conditions in which movements were allowed and/or long feedback tones were produced, we expected participants to develop associations between movement control (trajectory dynamics and energy expenditure), auditory feedback (the temporally unfolding shape of the envelope), and the target interonset interval (IOI) indicated by the metronome throughout the synchronization phase. In the continuation phase of the timing task, we expected that participants would rely on these learned auditory-motor patterns to maintain temporal regularity in their tapping (i.e., a perceptual-motor strategy). In conditions in which participants could not rely on learned auditory-motor temporal relationships, we expected that they would rely more on cognitive resources to explicitly count time (i.e., a timekeeper strategy), leading to impaired timing production under heightened cognitive load, because the timing mechanism requires the same cognitive resources as does the working memory task (cf. dual-task interference paradigm).

\section{Method}

Participants. Twenty-four right-handed participants (13 women) were recruited for the study, with an age range of 19-33 years. None of them had received formal musical or dance training. All reported not having any hearing problems or learning disorders. They participated freely and did not receive any financial compensation. Written informed consent was obtained prior to participation, and the Ethical Review Committee of the Faculty of Arts and Philosophy of Ghent University reviewed the experiment.

Materials and apparatus. The participants were asked to stand in front of a computer screen, which was set at a height of approximately $150 \mathrm{~cm}$. The screen could be tilted at different angles depending on a participant's height. A computer keyboard was placed in front of the computer screen at a height of approximately $100 \mathrm{~cm}$. The space bar of the keyboard was used to register the participants' finger taps. To cope with latency issues, we used an Empirisoft (New York, NY) DirectIN Millisecond Accurate keyboard (http://www.empirisoft.com/directinkb.aspx) in combination with the Psychophysics Toolbox Version 3 in MATLAB (Brainard, 1997; Kleiner, Brainard, \& Pelli, 2007) running on a MacBook Pro (Apple Inc., Cupertino, CA) laptop (2.53-GHz Intel [Santa Clara, CA] Core 2 Duo, 4-GB 1067-MHz DDR3, OSX 10.9.3). All sounds were played through Sennheiser (Wedemark, Germany) HD 201 headphones at a predefined comfortable volume level.

Stimuli. Each tap on the space bar of the computer keyboard triggered one piano tone, resulting in the production of a melody of 12 quarter notes, which was repeated after each round. The melody consisted of the pitches A4-G4-C5-D5-E5-C5-D5-E5C5-D5-A4-G4, as shown in Figure 1. Each piano tone was prepared in advance of the experiment according to a specific procedure. First, the piano tone was sampled from a MIDI synthesizer (AudioUnit DLS Music Device; Apple Inc.). The piano tone was characterized by a specific attack-decay-sustain-release amplitude envelope: a sharp attack, followed by a decay that lasted until around $300 \mathrm{~ms}$, then a sustain phase that was kept constant until around 1,500 ms, and finally a short release. Importantly, this waveform was multiplied with another envelope signal to obtain a natural-sounding piano tone, the decay and, by extension, the duration of which could be manipulated in a controlled way. This envelope signal consisted of a fast rising attack $(1 \mathrm{~ms})$ and an exponentially decreasing decay curve of which the decay rate could be changed, making a tone either longer or shorter. Within a trial, all tones were either short (short auditory feedback condition) or long (long auditory feedback condition). For the long tones, the decay curve decreased to $0.5 \%$ of the initial (peak) value after $1,100 \mathrm{~ms}$, thereby filling the complete duration of the target IOI of 1,100 ms (see the following section). For the short tones, the same percentage of decrease was reached after $300 \mathrm{~ms}$, thereby filling only a small portion of the target IOI.

Design and procedure. The experiment applied a dual-task paradigm (see Figure 2). The primary task-a timing-production task-was a typical synchronization-continuation task (Stevens, 1886; Wing \& Kristofferson, 1973). A metronome marked the beginning of the synchronization phase and produced equally spaced ticks with IOIs of 1,100 ms (cf. Maes et al., 2015). Participants were asked to listen to the first two metronome ticks and to start tapping the space bar of a computer keyboard from the

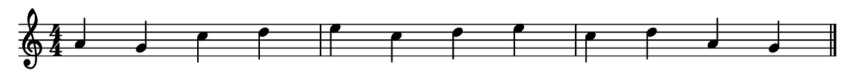

Figure 1. Score representation of the melody that was generated by the key presses in Experiment 1 (A4-G4-C5-D5-E5-C5-D5-E5-C5-D5A4-G4) 


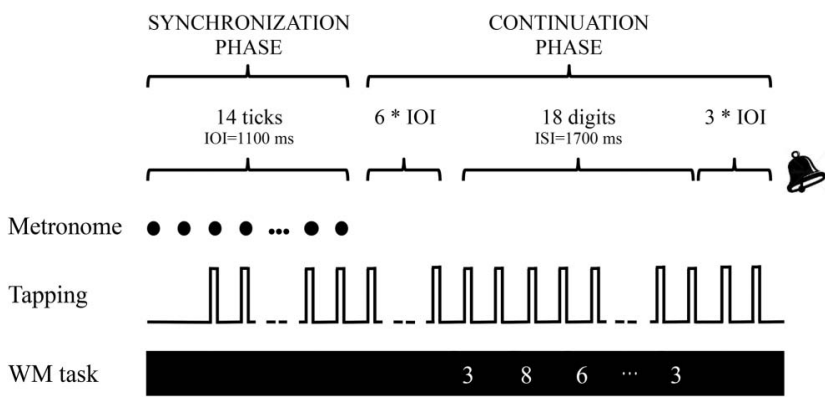

Figure 2. Schematic representation of the experimental design in Experiment 1 . IOI $=$ interonset interval; $\mathrm{WM}=$ working memory.

third tick onward, aligning each tone onset with each tick. The metronome ended after 14 ticks, and at that point the continuation phase commenced, during which participants were asked to continue tapping the space bar, matching the tempo indicated by the initial metronome as closely as possible. The continuation phase lasted $40.5 \mathrm{~s}$. The end of a trial was indicated by a bell sound.

The secondary task-a working memory task-was a digitswitch task (Krampe et al., 2010; Maes et al., 2015). This task involved both the storage and the manipulation of information in working memory (Baddeley \& Hitch, 1974). Digit strings of 18 digits in length were created, composed of single-digit numerals (1-9) ordered randomly on each trial, excluding immediate repetitions of the same digit. Participants were asked to count the number of switches from even to uneven digits and from uneven to even digits. The digit strings were presented on a computer screen placed in front of a participant. A new digit was presented every $1,700 \mathrm{~ms}$ and displayed until the next digit occurred. The first digit was displayed $6.6 \mathrm{~s}$ (equal to six taps) after the start of the continuation phase of the timing task to allow for tempo stabilization (Krampe et al., 2010). After the presentation of the last digit, participants could produce another three taps before the end of the trial. After each trial, participants were asked to report the two numbers, and they were immediately informed about the correct answers.

Movement type and auditory feedback were the two independent variables. Both related to the primary timing task. Movement type related to the way participants had to tap the space bar of the keyboard. We instructed participants either to continuously and smoothly move their whole arm up and down (one time) between two successive key presses in a way that felt comfortable for them (continuous movement type) or to leave their finger on the key at all times (discrete movement type). Auditory feedback related to the tones that were triggered by tapping the space bar. Tapping the space bar generated either a short or a long piano tone (as described earlier). The experimenters controlled whether a tone was long or short, meaning that the duration of the generated tone was independent of how participants tapped the space bar. The levels of the factors movement type and auditory feedback were fully crossed, leading to four conditions in which the timing task had to be performed, all in combination with the secondary working memory task. Participants were asked to perform three trials of each condition, leading to 12 trials in total. A complete trial took $55.4 \mathrm{~s}$.

Prior to the actual test phase of the experiment, participants were given time to practice both tasks in the different conditions. First, they practiced the timing task, then the working memory task, and finally both tasks in combination (as in the actual tests that followed). Participants needed to have four out of the last six trials correct to advance to the next task. For the timing task, a trial was considered correct when the mean of the ITIs did not exceed $\pm 15 \%$ of the target duration. The four correct trials for each participant in the training phase were used as baseline measures for the outcomes of the subsequent test phase. For the working memory task, a trial was considered correct when participants reported the correct numbers of odd-to-even and even-to-odd switches.

Data analysis: Linear mixed-effects (LME) modeling. ITIs were calculated from the registered space bar taps. We took into account only the ITIs that occurred during the presentation of the working memory task in the continuation phase. The duration of this presentation was $18 \times 1,700 \mathrm{~ms}$, approximating 28 ITIs (see Figure 2). ITIs that deviated $\pm 50 \%$ from the target IOI of $1,100 \mathrm{~ms}$ or 3 standard deviations from a trial's mean ITI were excluded from further analysis $(0.25 \%)$.

Taking general measures per trial-such as the mean, the standard deviation, or the related coefficient of variation of produced ITIs-implicitly assumes that ITIs do not evolve over time. However, when ITI values do evolve (drift) throughout the 28 ITIs produced within a trial, these general measures may lead to a distorted, or at least incomplete, view of a participant's performance. Therefore, we opted to use LME modeling, because this method allowed us to take into account ITIs as a function of time.

The data set of interest consisted of series of 28 ITIs for all 24 participants for each combination of movement type (discrete, continuous), auditory feedback condition (short, long), and task condition (single, dual). In this context, repeated measurements appear both through the combination of the factors movement type, auditory feedback, and task, because all individuals performed each combination of factor conditions, and through the longitudinal nature of the data, because ITI measurements were repeated (i.e., 28 times) over time for the same individuals. This implied a subject-specific dependence structure within the observations. Analysis of variance (ANOVA) and classical linear modeling were then no longer suited to account, respectively, for a proper modeling of evolution over time or for such a dependence structure. In contrast, mixed-effects models for longitudinal data analysis were suitable for our purposes, because they provided a unified framework to model time evolution while decomposing the effects of the factors on the response variable into a fixed part accounting for the main evolution pattern and into a random part modeling the subject-specific variability around the main pattern (Laird \& Ware, 1982). The underlying objective was thus to be able to accurately infer about covariates acting as fixed effects by properly decomposing the overall variability in a between-subject variability, modeling variations between individuals with respect to the main pattern, and a within-subject part, modeling variations within individual observations. In addition, mixed-effects models offered the advantage of naturally coping with missing data and nonbalanced grouping structure. Model fitting was carried out with the software R (Version 3.1.2), using the package nlme (Pinheiro \& Bates, 2000).

Basically, produced ITIs $(Y)$ of an individual $i(i=1, \ldots, 24)$ at time $t_{i j}(j=1, \ldots, 28), j$ representing the key press index, were modeled such that, given a combination of factors auditory feedback, movement type, and task, $Y_{i j}=\alpha_{i}+\beta_{i} t_{i j}+\varepsilon_{i j}$, where $\alpha_{i}$ and 
$\beta_{i}$ represent, respectively, the individual intercept and slope under the considered combination, and $\varepsilon_{i j}$ is an error term assumed to be normally distributed with zero mean and variance $\sigma^{2}$. Individual quantities could be further decomposed such that, given a combination of factors auditory feedback, movement type, and task,

$$
\alpha_{i}=\alpha+a_{i} \text { and } \beta_{i}=\beta+b_{i}
$$

where $\alpha$ represents a fixed intercept common to all individuals and depending on the levels of the factors auditory feedback, movement type, and task, whereas $\beta$ is a fixed slope parameter also depending on the factor levels. $\alpha$ and $\beta$ are usually referred to as fixed effects in the mixed-effects literature. In contrast, $\mathrm{a}_{i}$ and $\mathrm{b}_{i}$ are the respective individual random intercept and slope, usually referred to as random effects and standing for individual deviations around the average fitted line with respect to the factors.

\section{Results}

All effects are reported as significant at an alpha level of .05. Inference results in the mixed-effects framework are based on approximate $t$ tests and $F$ tests, as described in Verbeke and Molenberghs (2000, chap. 6). For the repeated-measure ANOVAsused for analyzing participants' working memory task performance-we tested for the assumption of sphericity using Mauchly's test. No corrections of degrees of freedom were required. Post hoc tests for interactions were conducted as $t$ tests, with alpha levels corrected for multiple comparisons using Bonferroni's method.

Working memory task: Comparison of correct trial proportions. For the participants' performances on the cognitive task in the dual-task condition, a two-way ANOVA was applied, with movement type (discrete, continuous) and auditory feedback (short, long) as within-subject factors to assess whether the average proportion of correct trials differed across conditions. Results showed no significant differences. On average, the proportion of correct trials was $.78($ SEM $=.026)$.

Timing task. After preliminary model-fitting steps, we considered a mixed-effects model with random intercepts with respect to subject and trial. Fixed effects and fixed interactions that revealed significance are reported in the ANOVA summary in Table 1 , and average profiles with respect to significant effects are represented in Figure 3.

Global ITI value: Intercept. For the global ITI value (i.e., intercept, concerning the parameter $\alpha$ in Equation 1), we found significant effects of auditory feedback and movement type as well as significant Auditory Feedback $\times$ Task, Movement Type $\times$ Task, and Auditory Feedback $\times$ Movement Type $\times$ Task interactions. Following up on these significant effects, an inspection of the estimated contrasts in Table 1 revealed that long auditory feedback and continuous movement type led participants to produce intervals that were generally closer to the target interval (1,100 ms) compared with short auditory feedback and discrete

Table 1

Significant Fixed Effects and Estimated Values for Fixed Effects in Experiment 1

\begin{tabular}{|c|c|c|c|c|}
\hline Fixed effect & & $d f$ & $F$ & $p$ \\
\hline \multicolumn{5}{|c|}{ Analysis of variance results for global significance of factors } \\
\hline \multicolumn{5}{|l|}{ Effect on intercept $(\alpha)$} \\
\hline Constant & & $(1,10,173)$ & $14,475.86$ & $<.001$ \\
\hline Feedback & & $(1,10,173)$ & 47.98 & $<.001$ \\
\hline Movement & & $(1,10,173)$ & 678.71 & $<.001$ \\
\hline Feedback $\times$ Task & & $(1,10,173)$ & 6.92 & .001 \\
\hline Movement $\times$ Task & & $(1,10,173)$ & 114.97 & $<.001$ \\
\hline Feedback $\times$ Movement $\times$ Task & & $(2,10,173)$ & 3.91 & .02 \\
\hline \multicolumn{5}{|l|}{ Effect on slope $(\beta)$} \\
\hline Time & & $(1,10,173)$ & 71.45 & $<.001$ \\
\hline Time $\times$ Task & & $(1,10,173)$ & 5.39 & .02 \\
\hline \multirow[t]{2}{*}{ Time $\times$ Feedback } & & $(1,10,173)$ & 4.01 & .045 \\
\hline & Estimate $(S D)$ & $d f$ & $t$ & $p$ \\
\hline \multicolumn{5}{|c|}{ Parameters estimates for fixed effects ${ }^{\mathrm{a}}$} \\
\hline \multicolumn{5}{|l|}{ Effect on intercept $(\alpha)$} \\
\hline Constant & $1,048.95(9.35)$ & 10,173 & 112.16 & $<.001$ \\
\hline Auditory Feedback (long) & $17.47(4.03)$ & 10,173 & 4.33 & $<.001$ \\
\hline Movement (continuous) & $13.53(3.46)$ & 10,173 & 3.91 & $<.001$ \\
\hline Auditory Feedback (short) $\times$ Task (dual) & $-13.07(3.82)$ & 10,173 & -3.42 & $<.001$ \\
\hline Auditory Feedback (long) $\times$ Task (dual) & $-31.01(3.81)$ & 10,173 & -8.14 & $<.001$ \\
\hline Movement (continuous) $\times$ Task (dual) & $21.97(3.95)$ & 10,173 & 5.57 & $<.001$ \\
\hline Auditory Feedback (long) $\times$ Movement (continuous) $\times$ Task $($ single $)$ & $-11.32(4.85)$ & 10,173 & -2.33 & .020 \\
\hline Auditory Feedback (long) $\times$ Movement (continuous) $\times$ Task (dual) & $4.15(2.69)$ & 10,173 & 1.54 & .12 \\
\hline \multicolumn{5}{|l|}{ Effect on slope $(\beta)$} \\
\hline Time & $-1.06(0.17)$ & 10,173 & -6.20 & $<.001$ \\
\hline Time $\times$ Task (dual) & $0.41(0.18)$ & 10,173 & 2.34 & .019 \\
\hline Time $\times$ Auditory Feedback (long) & $0.30(0.15)$ & 10,173 & 2.01 & .044 \\
\hline
\end{tabular}

${ }^{a}$ Short auditory feedback, discrete movement type, and single task served as reference levels. 


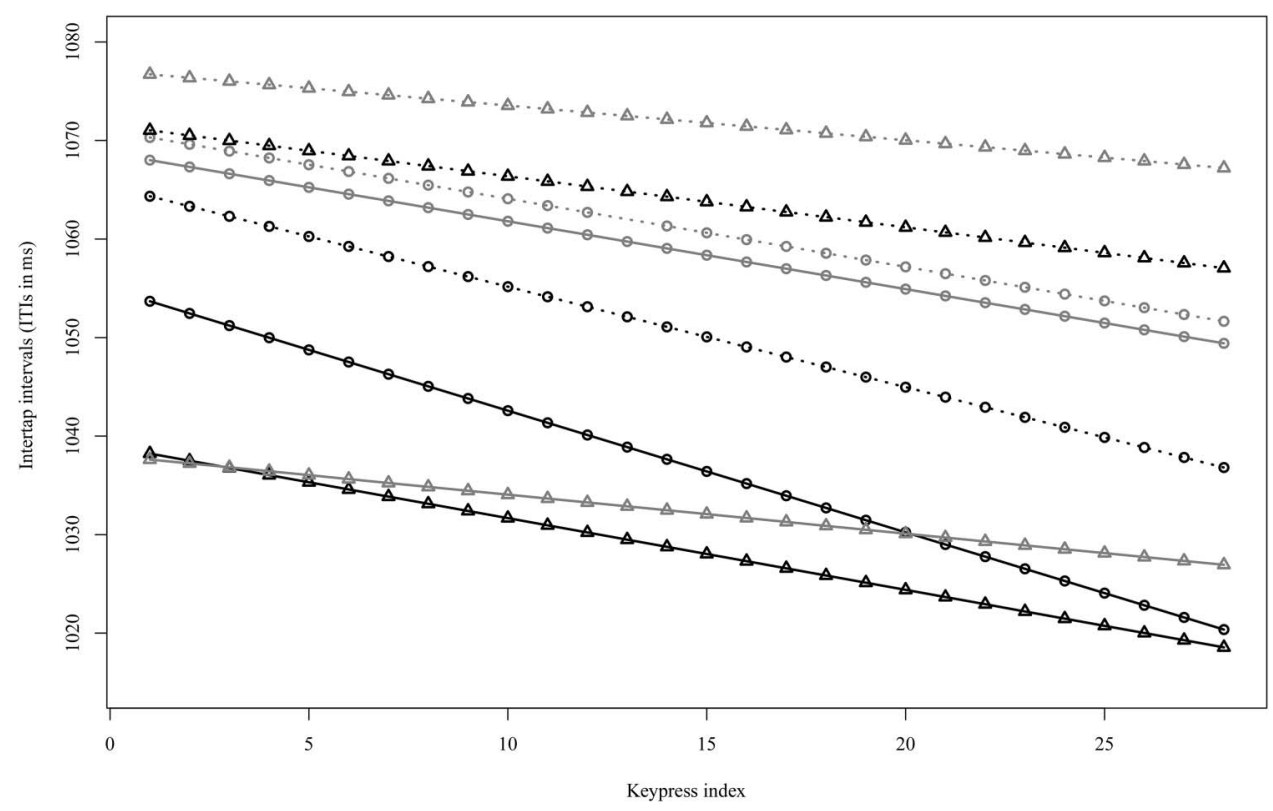

Figure 3. Average fitted profiles with respect to reported significant main effects and interactions in Experiment 1 . Short auditory feedback and long auditory feedback are respectively colored black and gray, and discrete movement type and continuous movement type are respectively represented by solid and dashed lines. Single and dual tasks are respectively represented by circle and triangle points.

movement type. In particular, produced intervals were closer to the target interval when going from the discrete to the continuous movement type in the dual-task setting. Compared with the singletask setting, in the dual-task setting, both auditory feedback conditions led to the production of intervals shorter, on average, than the target interval, which was mainly a result of poor performance when the movement type was discrete (see Figure 4). Finally, the significant Auditory Feedback $\times$ Movement Type $\times$ Task interaction indicated that the effects of auditory feedback and movement type were not the same in the single task as compared with the dual task. This is illustrated in Figure 4, where it can be observed that changing auditory feedback had a larger impact in

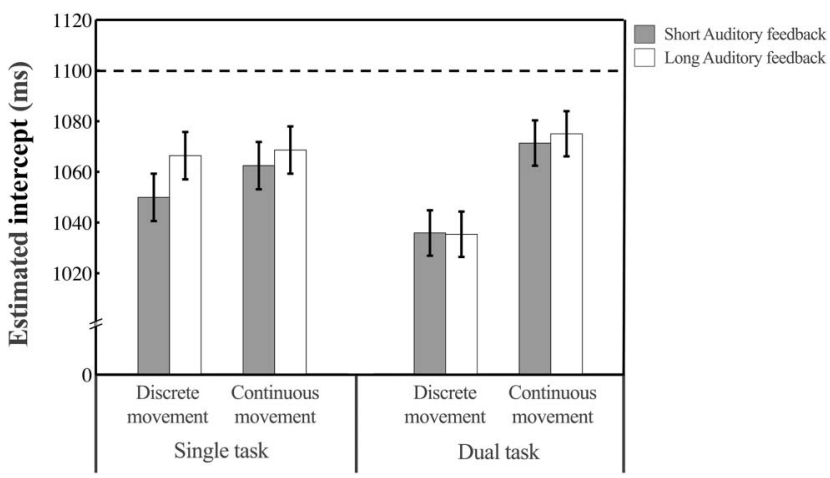

Figure 4. Estimated intercepts ( $\alpha$ s; see Equation 1) averaged across all participants in single-task and dual-task conditions, for discrete and continuous movement types, and for short and long auditory feedback in Experiment 1. Error bars represent standard errors of the mean. the single-task setting than in the dual-task setting, the main differences coming from the change in movement type.

Stability (drift): Slope. Concerning the evolution of ITIs over time (i.e., related to the parameter $\beta$ in Equation 1), we found a significant effect of time, indicating a global decrease of ITIs over time in all conditions. Moreover, this decrease was even more pronounced in the case of short auditory feedback and in the single-task setting: Parameter estimates for Auditory Feedback $\times$ Time and Dual Task $\times$ Time interactions were indeed significantly positive, indicating that observations were flattened in these auditory feedback and task conditions.

Variability: Estimated standard deviation (ms). For each trial, we calculated the estimated standard deviation as a measure of the variability of produced ITIs. The estimated standard deviation was calculated by first fitting a linear regression to the 28 ITI data points and then taking the sum of the squared differences between the data points and their projection on the fitted line divided by $n-2$, with $n=28$. We divided by $n-2$ because we estimated two parameters for the mean (intercept + slope), so $n-$ 2 gave an unbiased estimate. Averages of the estimated standard deviations for discrete and continuous movement types, for short and long auditory feedback, in the single- and dual-task conditions are shown in Figure 5. A three-way repeated-measures ANOVA was applied, with task (single, dual), movement type (discrete, continuous), and auditory feedback (short, long) as within-subject factors. A significant main effect of task was found, indicating a significant higher variability in the dual-task condition $(M=$ $46.98, S E M=2.54)$ compared with the single-task condition $(M=$ $41.17, S E M=2.62), F(1,23)=8.27, p=.009, \eta_{\mathrm{p}}^{2}=.27$. In addition, a significant main effect of movement type was found, with a higher average variability for the discrete movement type 


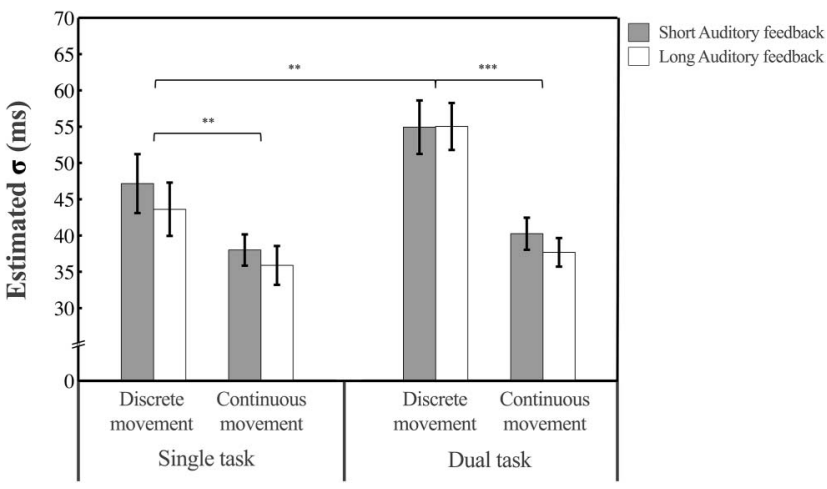

Figure 5. Estimated standard deviations $(\sigma \mathrm{s})$ averaged across all participants in single-task and dual-task conditions, for discrete and continuous movement types, and for short and long auditory feedback in Experiment 1. Error bars represent standard errors of the mean.

$(M=50.18, S E M=3.19)$ compared with the continuous movement type $(M=37.96, S E M=1.75), F(1,23)=38.57, p<.001$, $\eta_{\mathrm{p}}^{2}=.63$. Importantly, we found a significant Task $\times$ Movement Type interaction, $F(1,23)=6.24, p=.020, \eta_{\mathrm{p}}^{2}=.21$. Post hoc comparisons indicated that this interaction effect was driven by a significant increase in variability of the discrete movement type in the dual-task condition $(M=54.98, S E M=3.36)$ relative to the single-task condition $(M=45.38, S E M=3.73), t(23)=3.07, p=$ $.005, d=0.55$.

\section{Discussion}

Following up on Maes et al. (2015), we aimed to examine the relative contributions of motor control and auditory feedback to regular (suprasecond) interval production under cognitive load. To that end, participants performed an isochronous sequential interval-production task with and without an additional working memory task. Taken together, the results suggest that motor performance aspects contribute more to a reliable strategy for timing control under cognitive load compared with auditory feedback aspects. It was evidenced that, under heightened cognitive load, timing variability and global tapping tempo only increased significantly when participants were not allowed to perform movements between successive key presses. These findings suggest that the performance of arm movements between key presses may provide a reliable motor timing strategy to cope with situations of heightened cognitive load. To assess effects of auditory feedback on participants' timing performance, we manipulated the duration of the piano tones produced by key presses so that tones were either short or long (filling the complete duration of the target interval). We expected that the long tones would provide auditory information in relation to the target IOI such that temporal regularities could be realized by anchoring actions (i.e., taps of the key) in response to incoming perceptual information of the previously produced tone. Results indicated that produced interval durations were more stable - that is, there was less decrease in duration (drift) throughout the continuation phase-when tapping produced long tones compared with when it produced short tones. In addition, results showed that produced intervals were generally closer to the target interval when long tones were produced in contrast with when short tones were produced. However, the results showed that this effect was observed only when no additional cognitive load was applied. And, more particularly, results showed that the beneficial effect of long feedback tones when no additional cognitive load was applied was more pronounced when people were not allowed to make movements between successive key presses.

We consider two related reasons why auditory feedback- compared with motor performance aspects - could have had less impact on participants' timing performance under cognitive load. First, our results showed that produced intervals in the continuation phase were consistently shorter than the target interval. The shortening of the produced ITI had the consequence that the long tones did not exactly fit the interval-that is, they were sounding longer-which could have had the further consequence that their reference to the target interval became less obvious. Second, research has indicated that the ability to allocate attention to perceptual stimuli becomes worse under conditions of high load on cognitive control processes, such as working memory (Lavie, 2010; Lavie, Hirst, de Fockert, \& Viding, 2004), and that a visual perceptual load may equally lead to a considerable reduction of sensitivity in auditory detection (Raveh \& Lavie, 2015). Hence, the fact that the additional cognitive task used in this experiment increased working memory load as well as visual perception load could have been a reason participants failed to sufficiently allocate attention to the auditory feedback and, consequently, failed to use it as a reference in their timing performance. Therefore, in Experiment 2 , we introduced more obvious changes in the auditory feedback stimuli-more specifically, in the duration of the selfgenerated piano tones - to assess whether these could be more easily perceived and, consequently, affect people's timing control.

\section{Experiment 2}

In Experiment 2, we wanted to investigate the extent to which changes in self-generated auditory feedback could adapt people's temporal behavior. Again, we applied a synchronizationcontinuation tapping task. But now, throughout the continuation phase, we gradually changed the length of the produced piano tones, and we looked at the effects of this manipulation on people's temporal regularity. Previous research has suggested that during performance of a piano piece, the acoustic reverberation time of the room-determining the length of produced tones- has an influence on pianists' performed tempo (Bolzinger \& Risset, 1992; Kawai et al., 2013). Such effects on timing control indicate that people are sensitive to self-generated auditory feedback, on which they may rely to effectively control their timing performance. Here, we addressed this mechanism specifically from the perspective of prediction processes and sensorimotor adaptation (see the Auditory-Motor Adaptation section). We expected that our participants would develop an association between the duration of a tone-more specifically, the amplitude's decay envelope — and the target interval as a result of repeated experience in the synchronization phase of a trial. As a consequence, if participants were effectively sensitive to their auditory feedback, we expected that the amplitude's decay envelope of produced tones could function as a temporal reference for timing control in the continuation phase. Hence, aligning action (i.e., tapping the key) with a specific moment within the auditory decay pattern of the previous tone 
would enable participants to keep a stable pace without the need to explicitly calculate time (see the hourglass example in the Auditory-Motor Adaptation section). Use of such an auditorymotor strategy would entail that gradual lengthening or shortening of feedback tones would lead to predictable changes in tapping pace-respectively, a decrease and an increase in pace.

\section{Method}

Participants. The participants were the same as those in Experiment 1 .

Materials and apparatus. The materials and apparatus were the same as those used in Experiment 1.

Stimuli. Each tap on the space bar triggered a piano tone of the melody shown in Figure 1. The tones that were used were similar to the long tones used in Experiment 1. These tones filled the complete duration of the target interval; that is, the exponentially decaying amplitude's envelope was reduced to $0.5 \%$ of the initial (peak) value at the target ITI of 1,100 ms. However, in some conditions, the tones' decay envelope was gradually altered so that a similar percentage of decay was obtained $233 \mathrm{~ms}$ earlier (867 $\mathrm{ms})$ or later $(1,333 \mathrm{~ms})$, thus making tones respectively shorter and longer (see Figure 6, top middle and bottom panels). More details about this alteration are provided in the following section.

Design and procedure. All participants completed Experiment 1 before Experiment 2. There was a small break between experiments of about $10 \mathrm{~min}$, during which participants could relax a bit and have something to eat or drink. We applied a dual-task paradigm consisting of a timing task and a working memory task. Movement type and auditory feedback were the two independent variables. Participants had to either perform up-and-down arm movements between successive key presses (continuous movement type condition) or keep their finger on the key to avoid any movement between key presses (discrete movement type condition). The variable auditory feedback related to the specific alteration of the tones' amplitude envelopes (equal, longer, shorter). Tone envelopes remained unchanged throughout the synchronization phase and the first six taps of the continuation phase so that participants could learn the relationship between movement pattern, auditory feedback, and the passage of the target temporal interval. Hence, the alteration of the tones' amplitude envelopes started as soon as the first digit of the working memory task appeared. The presentation of the working memory task, which
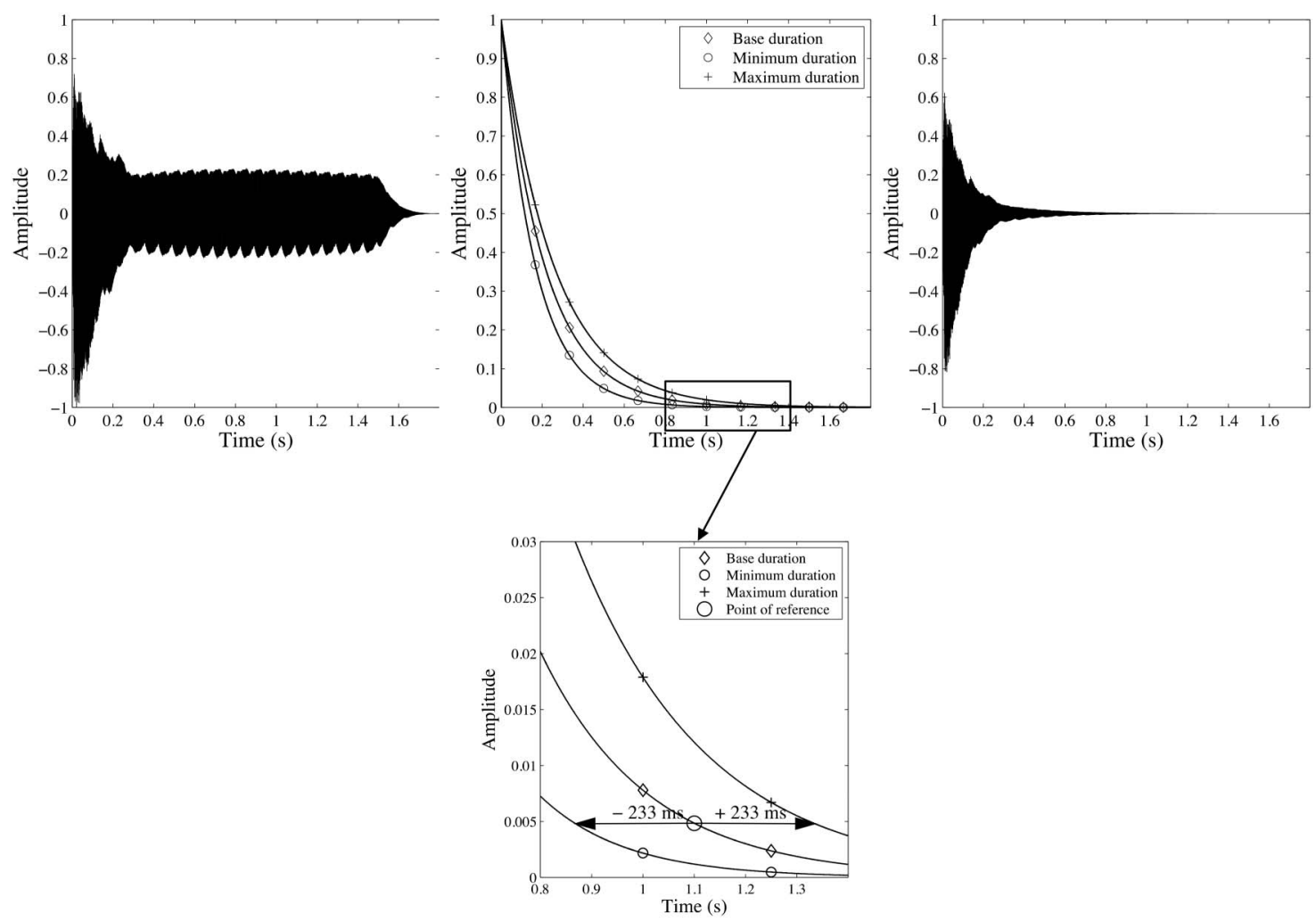

Figure 6. Auditory feedback manipulations in Experiment 2. Top left: waveform representation of a piano tone sampled from a MIDI synthesizer. Top middle: envelope signals, with the one marked with diamond points being the envelope used throughout the equal auditory feedback condition, the one marked with circle points being the envelope signal that was gradually shifted toward throughout the shorter auditory feedback condition, and the one marked with plus points being the envelope signal that was gradually shifted toward throughout the longer auditory feedback condition. Top right: waveform representation of the multiplication of the original piano tone with an amplitude's envelope (here, the envelope signal marked with diamond points in the top middle panel). Bottom: detail of the amplitude's envelopes at the time corresponding to the target interonset interval $(1,100 \mathrm{~ms})$. 
was performed in all conditions, lasted $30.6 \mathrm{~s}(18 \times 1,700 \mathrm{~ms})$, approximating 28 ITIs. As explained in the previous section, tones were gradually shortened or lengthened throughout this series of 28 ITIs to realize a duration change of $233 \mathrm{~ms}$ after 28 ITIs. The experiment was organized into four blocks that were counterbalanced across participants. Each block started with a trial in which no alteration of the tones' amplitude envelopes occurred. The remaining three (identical) trials applied either a shortening or lengthening of the tone duration, crossed with both levels of the movement type variable, leading to four blocks. The experiment took about $15 \mathrm{~min}$, without taking into account breaks between trials.

\section{Results}

Working memory task: Comparison of correct trial proportions. A two-way ANOVA was applied, with movement type (discrete, continuous) and auditory feedback (equal, longer, shorter) as within-subject factors. We found a main effect of movement, $F(1,23)=4.46, p=.046$. On average, the proportion of correct trials for the continuous movement type $(M=.87$, $S E M=.03)$ was significantly higher than that for the discrete movement type $(M=.81, S E M=.04)$.

Timing task. Similar to Experiment 1, we used LME models to perform a longitudinal analysis of the data. However, in Experiment 2, we were particularly interested in the evolution of individual ITIs over time in relation to the auditory feedback condition. Raw average profiles per auditory feedback condition are represented in Figure 7. In a first approach, data were normalized by centering them individually with respect to their intercept to focus on time-dependent effects of the auditory feedback manipulations on ITI values. Note that this centering did not affect the ITIs' evolution over time (i.e., the slope). Regarding our main objective, following preliminary model-fitting steps, the fixed in- tercept $\alpha$ in Equation 1 was assumed to be a common intercept for all factors' levels and all individuals, whereas the fixed slope $\beta$ depended on the auditory feedback condition (equal, longer, shorter). Random intercepts stood for individual deviations around average profile intercepts and modeled the individual variation of the responses regarding movement type and trial index, respectively. Random intercepts were assumed to be normally distributed, with zero mean and a nonstructured covariance matrix. Estimates of the model parameters are reported in Table 2. Main patterns by auditory feedback condition are represented in Figure 7 , and examples of individual profile fittings (best unbiased linear predictions) are represented in Figure 8.

Stability (drift): Slope. The obtained results revealed a significant decrease of ITI values over time for all auditory feedback conditions. A further analysis of fixed-effect contrasts enabled assessment of specific effects of shortening and lengthening the tones' durations: Values in Table 2 indicate that the gradual shortening of the tones' durations throughout a trial significantly shortened the ITIs over time (Time $\times$ Auditory Feedback [shorter]). In contrast, lengthening the tones' durations throughout a trial did not significantly flatten the evolution of ITI values over time compared with the reference equal auditory feedback (Time $\times$ Auditory Feedback [longer]).

Global ITI value: Intercept. It is worth noting that a similar analysis on the nonnormalized data set yielded effects of movement type and auditory feedback on the ITIs' intercepts while conclusions regarding the evolution of ITIs over time with respect to auditory feedback still held. This analysis was performed by making the fixed intercept $\alpha$ in Equation 1 dependent on movement type and auditory feedback conditions in the fixed part of the model. Results revealed that produced intervals were, in general, closer to the target interval when using the continuous movement type compared with the discrete movement type, $t(10,439)=5.28$,

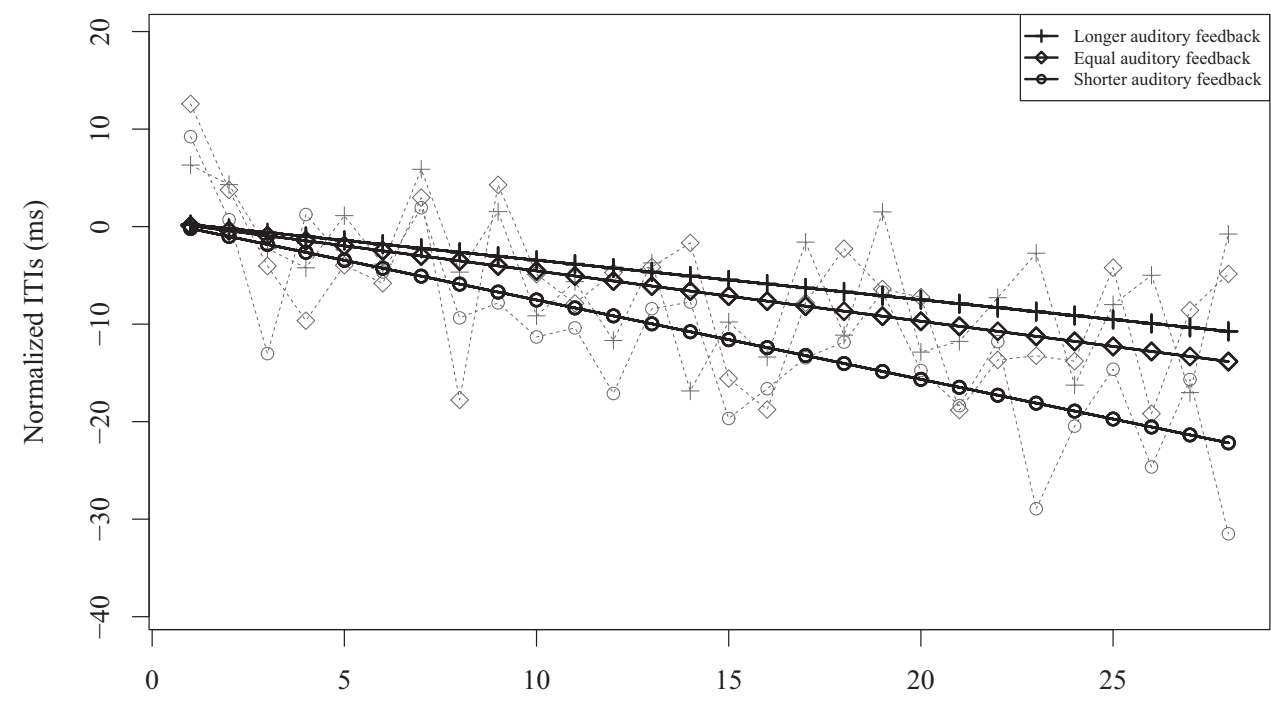

Keypress index

Figure 7. Auditory feedback-specific profiles plotted against keypress index. Raw profiles averaged across individuals (thin lines) and linear fittings (bold lines) are represented by auditory feedback condition. ITIs $=$ intertap intervals (in $\mathrm{ms}$ ). 
Table 2

Significant Fixed Effects and Estimated Values for Fixed Effects on the Normalized Intertap Interval Data Set in Experiment 2

\begin{tabular}{|c|c|c|c|c|}
\hline Fixed effect & & $d f$ & $F$ & $p$ \\
\hline \multicolumn{5}{|c|}{ Analysis of variance results for global significance of factors } \\
\hline \multicolumn{5}{|c|}{ Effect on intercept $(\alpha)$} \\
\hline Constant & & $(1,10,442)$ & 13.38 & $<.001$ \\
\hline \multicolumn{5}{|l|}{ Effect on slope $(\beta)$} \\
\hline Time & & $(1,10,442)$ & 80.59 & $<.001$ \\
\hline \multirow[t]{2}{*}{ Time $\times$ Auditory Feedback } & & $(2,10,442)$ & 16.21 & $<.001$ \\
\hline & Estimate $(S D)$ & $d f$ & $t$ & $p$ \\
\hline \multicolumn{5}{|c|}{ Parameters estimates for fixed effects ${ }^{\mathrm{a}}$} \\
\hline \multicolumn{5}{|l|}{ Effect on intercept $(\alpha)$} \\
\hline Constant & $0.613(2.30)$ & 10,442 & 0.27 & .79 \\
\hline \multicolumn{5}{|l|}{ Effect on slope $(\beta)$} \\
\hline Time & $-0.516(0.086)$ & 10,442 & -6.00 & $<.001$ \\
\hline Time $\times$ Auditory Feedback (longer) & $0.110(0.084)$ & 10,442 & 1.32 & .19 \\
\hline Time $\times$ Auditory Feedback (shorter) & $-0.298(0.084)$ & 10,442 & -3.56 & $<.001$ \\
\hline
\end{tabular}

${ }^{a}$ Equal auditory feedback served as the reference level.

$p<.001$. A similar conclusion was highlighted in Experiment 1. The results also indicated significantly shorter ITIs for the equal auditory feedback, with differences between longer and equal, $t(10,439)=4.04, p<.001$, and shorter and equal, $t(10,439)=$ $5.99, p<.001$, ITIs.

Individual 7

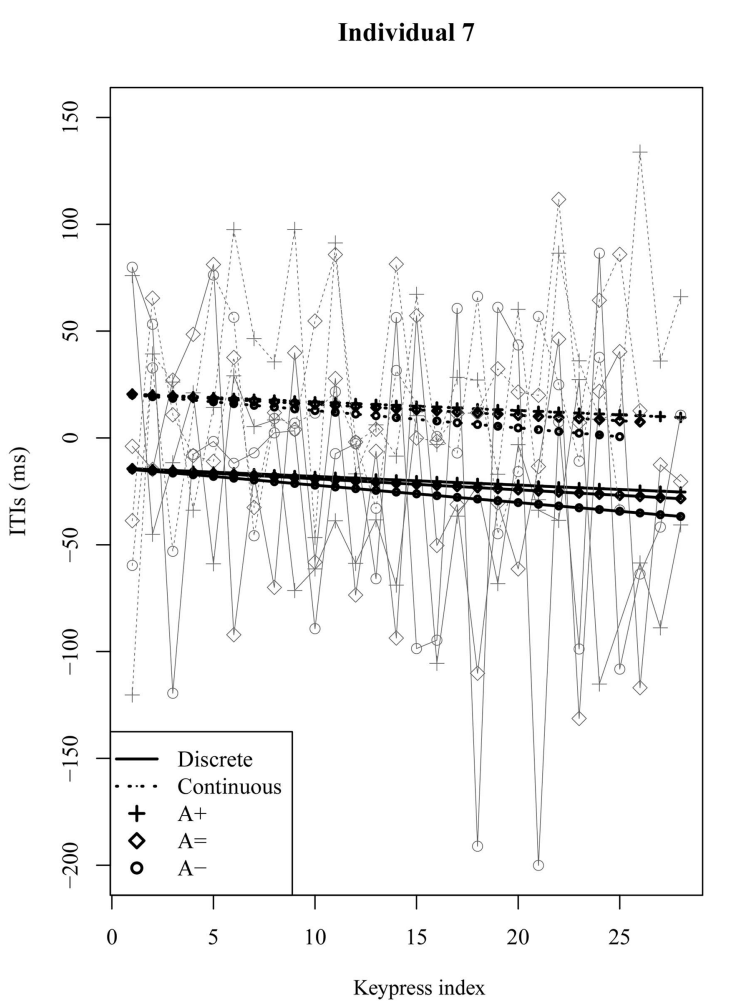

Variability: Estimated standard deviation (ms). Similar to Experiment 1, we calculated the average least-squares standard deviation per condition. A two-way repeated-measures ANOVA was applied, with movement type (discrete, continuous) and auditory feedback (equal, longer, shorter) as within-subject factors. A

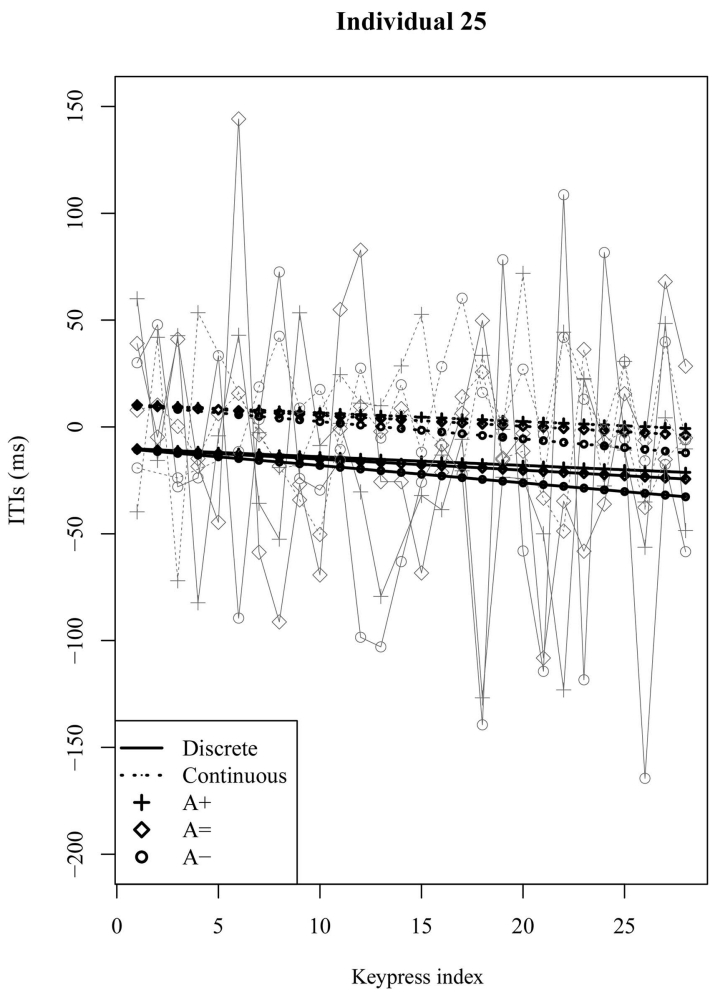

Figure 8. Examples of two individual fittings plotted by movement type and auditory feedback condition (A; equal, longer, and shorter marked by diamond, plus, and circle points, respectively). Random effects act as deviations on individual intercepts regarding movement type condition within one individual. ITIs = intertap intervals (in ms). 
significant main effect of movement type was found, indicating a significant increase in variability of the discrete movement type $(M=53.59, S E M=3.63)$ compared with the continuous movement type $(M=38.40, S E M=2.25), F(1,23)=54.09, p<.001$, $\eta_{\mathrm{p}}^{2}=.70$.

\section{Discussion}

Using a synchronization-continuation tapping paradigm, Experiment 2 aimed to investigate whether a gradual lengthening or shortening of self-generated piano tones could influence participants' tapping pace during continuation tapping. Results indicated a general tendency to speed up tapping pace throughout the continuation phase. Interesting, however, was the finding that a gradual shortening of feedback tones throughout the continuation phase allowed people to gradually speed up their tapping pace significantly more compared with when no manipulations were applied to the feedback tones. In contrast, the gradual lengthening of feedback tones did not lead to any significant changes in participants' interval-production rate with respect to the conditions in which feedback tones were not manipulated. A possible explanation of this finding relates to previously discussed sensorimotor adaptation mechanisms (see the Auditory-Motor Adaptation section). Throughout the synchronization phase, participants were expected to develop an association between the duration of a produced tone and the target interval that needed to be produced; in this case, the tone precisely fitted the target interval. In that regard, the offset of the tone could function as a perceptual point of reference (see Figure 6) to which actions (i.e., taps of a key) needed to be aligned. Accordingly, when the target tempo was kept constant, the auditory outcome of a key press-more particularly, the tone offset-was expected to match the onset of the next key press. However, when the duration of a tone was shortened, this expectancy was violated, and we hypothesized that actions would be adapted correspondingly. The results indeed showed that participants shifted their production of tone onsets toward the perceptual reference point, leading to a gradual increase in intervalproduction rate. In contrast, the lengthening of the tone's duration throughout a trial resulted not in a gap but, rather, in a small increase of the amplitude with respect to the original amplitude of the reference point. Presumably, this difference was too small to be perceived as an error, with the result that no adaptation of motor responses was applied by the participants. In addition, along with time-dependent (drift) effects on ITI values, results indicated that shorter intervals were produced in the equal auditory feedback condition. The finding that the intercept of equal auditory feedback was lower may have been attributable to the fact that the equal auditory feedback condition was always the first in a block, so changes in conditions from block to block required participants to get acquainted with those changes for a period.

Looking at movement type, similar effects as in Experiment 1 were found. Results showed that, in general, produced intervals were closer to the target interval, and the durations of the produced intervals exhibited less variability when participants were allowed to make movements between successive key presses. Interestingly, in contrast to Experiment 1, we also found a main effect of movement type on performance in the working memory task; participants made significantly more errors on the working memory task when no movements were allowed between successive key taps. This finding is in line with our hypothesis that both the working memory task and the discrete tapping task relied on similar cognitive resources, with bidirectional interference as a consequence. This replicates the results of Maes et al. (2015) and of other studies (Brown, 2006; Krampe et al., 2010; Rattat, 2010). The absence of any effect of movement type on participants' performance in the working memory task in Experiment 1 could have been a result of participants not yet being fully acquainted with the task and therefore making more errors in general, as indicated by a comparison of correct trial proportions in Experiment $1(M=.78, S E M=.026)$ and Experiment $2(M=.84$, $S E M=.035)$.

\section{General Discussion}

Following up on Maes et al. (2015), the present study aimed to investigate the relative contributions of motor performance aspects and self-generated auditory feedback to regular interval production under cognitive load. Concerning the role of motor performance aspects, results of both Experiment 1 and Experiment 2 suggested that an additional working memory task had a significantly higher impact on regular interval production when no movements were allowed between successive key presses compared with when movements were allowed, leading to lower accuracy and higher variability of produced IOIs. This finding indicates that timing performance in the absence of body movements between successive key presses relied on cognitive resources shared with the performance of the working memory task. This confirms the results of Maes et al. (2015) and other research arguing that the temporal control of discrete rhythmic body movements relies on an event-based timing system that depends on explicit computation of the passage of time (Braun Janzen et al., 2014; Delignières et al., 2004; LaRue, 2005; Robertson et al., 1999; Studenka et al., 2012; Torre \& Balasubramaniam, 2009; Zelaznik et al., 2002, 2008). In contrast, the fact that regular interval production was significantly better when participants were able to perform continuous arm movements between successive key presses suggests that timing control is regulated, at least in part, by sensorimotor processes, presumably capitalizing on the motor system's dynamics (emergent timing).

An important aim of this study was to assess the extent to which the duration of auditory feedback tones (here, piano tones) could support and guide regular interval production. Previous research has demonstrated that the way individuals synchronize with an external auditory-pacing signal is influenced by the continuity of that signal. For instance, Varlet et al. (2012) showed that synchronization to discrete and continuous stimulus rhythms resulted, respectively, in a delay and in anticipation in tappers' movement responses. In addition, Rodger and Craig (2011) found that smaller synchronization errors occurred when movements were synchronized to (isochronous) discrete sounds but that movements exhibited less variability when synchronized to continuous sounds. However, the question is whether these findings extend to selfgenerated feedback. Manning and Schutz (2015) showed that synchronized tapping to an isochronous beat is more accurate when people receive auditory feedback on their tapping. In contrast, other research has suggested that self-generated auditory feedback adds little to the information already provided by proprioception for performance accuracy, measured by negative asyn- 
chronies (Stenneken, Prinz, Cole, Paillard, \& Aschersleben, 2006). Similar ambiguities have been found in studies on regular tapping performance at a self-selected tempo or a target tempo. A study by Kolers and Brewster (1985) suggested that regular tapping at a specific target tempo is less variable when tappers receive selfgenerated auditory feedback (10-ms tones) compared with visual (light) or tactile (vibration) feedback. Also, van Vugt and Tillmann (2015) demonstrated that in learning to tap a sequence of keystrokes, regularity was significantly improved when taps generated tones instantaneously compared with when tones were presented after a random delay or no tones were presented. However, in another study, Roche, Wilms-Floet, Clark, and Whitall (2011) investigated-using a bilateral self-selected tapping task-the extent to which auditory feedback (generated by hard plastic caps placed on the tips of fingers tapping on a table) contributed to the overall motor response in terms of timing consistency, coordination accuracy, and coordination ability. They found that the removal of auditory information did not alter tapping performance in any of the variables.

However, in all of the foregoing studies, self-generated feedback was discrete (i.e., distinct auditory pulses or sounds separated by silence). In the current study, we investigated the extent to which piano tones filling the complete duration of the target interval could support and guide timing control. We expected that auditory patterns - here, the temporal unfolding of a tone's amplitude envelope - could provide a temporal reference for regular interval production. In Experiment 1, we found that, in general, when key tapping generated long tones, less tempo drift occurred in the continuation phase compared with when short tones were generated. In addition, participants kept a tapping tempo that was closer to the target tempo when key tapping generated long piano tones compared with when it generated short piano tones. However, this effect was present only in the absence of an additional working memory task, suggesting that the auditory temporal reference was presumably too subtle to be grasped under conditions of an increased cognitive and perceptual load induced by the working memory task (Lavie, 2010; Lavie et al., 2004; Raveh \& Lavie, 2015). In Experiment 2, we introduced more obvious changes to the piano tones throughout the continuation phase; we gradually lengthened or shortened piano tone durations up to 233 $\mathrm{ms}$. The most interesting finding was that shortening the duration of tones throughout the continuation phase made people shorten the intervals between produced onsets correspondingly. This finding is in agreement with previous research. For instance, Furuya and Soechting (2010) found that when pianists played particular measures of musical pieces (with IOIs $=333 \mathrm{~ms}$ ), delaying the auditory feedback of one tone shortened the subsequent produced interkeystroke intervals and finger-key contact durations. Another example is a study by Bolzinger and Risset (1992) investigating the influence of room acoustics on piano performances. Their results suggested that pianists play slower (i.e., tempo decreases) when reverberation time increases.

Finally, we refer to a review of studies demonstrating the role of auditory feedback in music performance by Pfordresher (2006). This study is of particular interest because it discussed the underlying principles of how auditory feedback influences many characteristics of a music performance. These principles are based on the interplay between action and perception and, more particularly, on existing correspondences between actions and the related auditory outcomes of these actions. Most studies that have investigated the role of auditory feedback have capitalized on a perturbation paradigm (cf. altered auditory feedback), whereby mismatches are introduced between actions and expected auditory feedback, typically along the dimensions of time and pitch. Alteration of auditory feedback has been shown to engender disruptions in various performance aspects. One explanation for this finding is based on the existence of shared representations between the perception of sensory feedback and the planning of actions (Hommel, Müsseler, Aschersleben, \& Prinz, 2001). It is suggested that these shared representations are incremental, in the sense that they incorporate information about past, present, and future events at each time instance (cf. the range model of Palmer \& Pfordresher, 2003). Consequently, hearing auditory events that correspond to other sequence positions may disrupt action planning (Pfordresher, 2006; Pfordresher \& Palmer, 2006). This notion underlines the importance of the coupling of action and perception in (musical) timing performance. However, as Pfordresher acknowledged, it does not address the usefulness of auditory feedback in, for example, timing control.

In the present research, we focused particularly on how selfgenerated auditory feedback may provide temporal patterns that function as a temporal reference on which people can rely to coordinate their timing. We speculated that auditory information may contain temporal patterns of dynamic change that can be used to index time. Further, we suggested two mechanisms that could account for this principle. First, we emphasized the importance of associative learning processes. Through the repeated experience of a performed action generating a particular auditory outcome (here, a particular tone's amplitude envelope) in relation to a temporal reference (here, an auditory metronome), one gradually learns how the changing shape of the tone's envelope indexes, or encodes, temporal progress (Addyman et al., 2011). Consequently, keeping a stable pace can be accomplished by properly aligning actions to this auditory pattern (cf. auditory scaffolding [Conway, Pisoni, \& Kronenberger, 2009] and auditory latching [DeNora, 2000]). Timing may then be realized in a perceptual manner, without the need to explicitly compute time. Second, we outlined the working of an automatic adaptation mechanism. Once an action and its auditory outcome are integrated in relation to a temporal reference, altering the auditory outcome-more particularly, making a tone shorter-led to a discrepancy between the expected and the actual tone duration. As a result, we observed that participants adapted their production rates to reduce this prediction error.

The main findings of both experiments are important, because they outline useful perceptual-motor strategies to improve timing performance under cognitive load. These strategies concern the "outsourcing" of cognitive functions to the human sensory and sensorimotor system to optimize task performance, efficiency, and productivity. This strategy makes sense in view of the stunning sensory and sensorimotor capabilities of humans in relation to their rather limited cognitive capacities (Moravec, 1988). Heightened cognitive load makes a clock-based timing strategy (cf. event-based timing and the timekeeper approach) less efficient as cognitive resources, such as working memory and attention, get "double-booked." Previous research has indicated that the timing strategy that people 
use depends on task constraints and personal factors (Braun Janzen et al., 2014). However, we do not wish to claim that clock-based and perceptual-motor timing strategies are mutually exclusive. Increasing evidence demonstrates that clockbased and nonclock-based timing strategies may coexist in performance of timing tasks (Braun Janzen et al., 2014; Hogan \& Sternad, 2007; Repp \& Steinman, 2010; Studenka, 2015).

The findings of this study provide an argument to allow and use body movements in music and musical instrument instruction practice (Nijs \& Leman, 2014). In addition, knowledge of the fundamental mechanisms underlying spontaneous motor adaptation may be applied in diverse areas of practice and research, such as sports (Lauber \& Keller, 2014) and rehabilitation (Rosati, Rodà, Avanzini, \& Masiero, 2013). In these domains, it is often useful to be able to guide motor behavior toward specific goals (faster/ slower, smaller/bigger, lighter/heavier, more fluent/more rigid, higher/lower, etc.). The present study introduces an innovative strategy - based on associative learning, prediction, and automatic error-correction mechanisms-to use sonic interactions for the purpose of motor adaptation. However, this requires an intense, interdisciplinary collaboration between musicologists, psychologists, musicians/music producers, engineers, and people from within the fields of sports and rehabilitation research and practice (Leman, 2007).

\section{Conclusion}

We investigated how sensory and sensorimotor information can support temporal control under heightened cognitive load. For that purpose, we applied a dual-task paradigm, using participants without musical training. In Experiment 1, we found that participants produced temporal intervals that were more accurate (i.e., closer to the target tempo) and more consistent (i.e., less variable) when they were allowed to perform movements between successive key presses. In addition, we found that when key tapping produced long piano tones, people exhibited less tempo drift and were, in the absence of additional cognitive load, closer to the target tempo. In Experiment 2, we applied alterations to the self-generated auditory feedback; tone durations were gradually increased or decreased throughout the continuation phase. It was found that gradually decreasing tone durations significantly sped up the interval-production rate of the participants. This suggests that self-generated auditory feedback may be used as a temporal reference, because actions become aligned with temporal cues in the auditory information. The findings of this study demonstrate that timing is not solely a matter of explicitly computing time but, rather, that people may capitalize on sensory and sensorimotor processes in interaction with the external environment to regulate timing production. On the basis of the particular design of the study-encompassing a synchronizationcontinuation paradigm and a motor-adaptation paradigm-we suggested some mechanisms that could account for the observed results, with a primary role for associative learning, prediction, and automatic error-correction mechanisms. These mechanisms could be further explored in a wide range of research and practice areas in which motor adaptation is of interest, such as sports or motor rehabilitation.

\section{References}

Adams, J. A. (1971). A closed-loop theory of motor learning. Journal of Motor Behavior, 3, 111-150. http://dx.doi.org/10.1080/00222895.1971 .10734898

Addyman, C., French, R. M., Mareschal, D., \& Thomas, E. (2011). Learning to perceive time: A connectionist, memory-decay model of the development of interval timing in infants. In L. Carlson, C. Hoelscher, \& T. F. Shipley (Eds.), Expanding the space of cognitive science: Proceedings of the 33rd Annual Conference of the Cognitive Science Society (pp. 354-359). Austin, TX: Cognitive Science Society.

Allman, M. J., Teki, S., Griffiths, T. D., \& Meck, W. H. (2014). Properties of the internal clock: First- and second-order principles of subjective time. Annual Review of Psychology, 65, 743-771. http://dx.doi.org/ 10.1146/annurev-psych-010213-115117

Baddeley, A. D., \& Hitch, G. (1974). Working memory. In G. H. Bower (Ed.), The psychology of learning and motivation: Advances in research and theory (Vol. 8, pp. 47-89). New York: Academic Press.

Bishop, L., Bailes, F., \& Dean, R. T. (2014). Performing musical dynamics: How crucial are musical imagery and auditory feedback for expert and novice musicians? Music Perception, 32, 51-66. http://dx.doi.org/ 10.1525/mp.2014.32.1.51

Bolzinger, S., \& Risset, J. C. (1992). A preliminary study on the influence of room acoustics on piano performance. Journal de Physique IV, 02, C1-93-96-C1. http://dx.doi.org/10.1051/jp4:1992116

Brainard, D. H. (1997). The Psychophysics Toolbox. Spatial Vision, 10 433-436. http://dx.doi.org/10.1163/156856897X00357

Braun Janzen, T., Thompson, W. F., Ammirante, P., \& Ranvaud, R. (2014). Timing skills and expertise: Discrete and continuous timed movements among musicians and athletes. Frontiers in Psychology, 5, 1482. http:// dx.doi.org/10.3389/fpsyg.2014.01482

Bravi, R., Del Tongo, C., Cohen, E. J., Dalle Mura, G., Tognetti, A., \& Minciacchi, D. (2014). Modulation of isochronous movements in a flexible environment: Links between motion and auditory experience. Experimental Brain Research, 232, 1663-1675. http://dx.doi.org/ 10.1007/s00221-014-3845-9

Brown, S. W. (1997). Attentional resources in timing: Interference effects in concurrent temporal and nontemporal working memory tasks. Perception \& Psychophysics, 59, 1118-1140. http://dx.doi.org/10.3758/ BF03205526

Brown, S. W. (2006). Timing and executive function: Bidirectional interference between concurrent temporal production and randomization tasks. Memory \& Cognition, 34, 1464-1471. http://dx.doi.org/10.3758/ BF03195911

Church, R. M. (1984). Properties of the internal clock. In J. Gibbon \& L. Allan (Eds.), Timing and time perception: Annals of the New York Academy of Sciences (Vol. 423, pp. 566-582). New York: New York Academy of Sciences. http://dx.doi.org/10.1111/j.1749-6632.1984 .tb23459.x

Conway, C. M., Pisoni, D. B., \& Kronenberger, W. G. (2009). The importance of sound for cognitive sequencing abilities: The auditory scaffolding hypothesis. Current Directions in Psychological Science, 18, 275-279. http://dx.doi.org/10.1111/j.1467-8721.2009.01651.x

Delignières, D., Lemoine, L., \& Torre, K. (2004). Time intervals production in tapping and oscillatory motion. Human Movement Science, 23, 87-103. http://dx.doi.org/10.1016/j.humov.2004.07.001

DeNora, T. (2000). Music in everyday life. New York: Cambridge University Press. http://dx.doi.org/10.1017/CBO9780511489433

Elliott, M. T., Wing, A. M., \& Welchman, A. E. (2014). Moving in time: Bayesian causal inference explains movement coordination to auditory beats. Proceedings of the Royal Society B: Biological Sciences, 281 , 20140751. http://dx.doi.org/10.1098/rspb.2014.0751

Feng, Y., Gracco, V. L., \& Max, L. (2011). Integration of auditory and somatosensory error signals in the neural control of speech movements. 
Journal of Neurophysiology, 106, 667-679. http://dx.doi.org/10.1152/jn .00638 .2010

Finney, S. A., \& Palmer, C. (2003). Auditory feedback and memory for music performance: Sound evidence for an encoding effect. Memory \& Cognition, 31, 51-64. http://dx.doi.org/10.3758/BF03196082

Fortin, C., \& Breton, R. (1995). Temporal interval production and processing in working memory. Perception \& Psychophysics, 57, 203-215. http://dx.doi.org/10.3758/BF03206507

Friston, K., Kilner, J., \& Harrison, L. (2006). A free energy principle for the brain. Journal of Physiology-Paris, 100, 70-87. http://dx.doi.org/ 10.1016/j.jphysparis.2006.10.001

Furuya, S., \& Soechting, J. F. (2010). Role of auditory feedback in the control of successive keystrokes during piano playing. Experimental Brain Research, 204, 223-237. http://dx.doi.org/10.1007/s00221-0102307-2

Gibbon, J., Church, R. M., \& Meck, W. H. (1984). Scalar timing in memory. In J. Gibbon \& L. Allan (Eds.), Timing and time perception: Annals of the New York Academy of Sciences (Vol. 423, pp. 52-77). New York: New York Academy of Sciences. http://dx.doi.org/10.1111/ j.1749-6632.1984.tb23417.x

Gibson, J. (1979). The ecological approach to visual perception. Boston: Houghton Mifflin.

Heyes, C. M., \& Ray, E. D. (2000). What is the significance of imitation in animals? Advances in the Study of Behavior, 29, 215-245. http://dx doi.org/10.1016/S0065-3454(08)60106-0

Hickok, G., Houde, J., \& Rong, F. (2011). Sensorimotor integration in speech processing: Computational basis and neural organization. Neuron, 69, 407-422. http://dx.doi.org/10.1016/j.neuron.2011.01.019

Hogan, N., \& Sternad, D. (2007). On rhythmic and discrete movements: Reflections, definitions and implications for motor control. Experimental Brain Research, 181, 13-30. http://dx.doi.org/10.1007/s00221-0070899-y

Hommel, B., Müsseler, J., Aschersleben, G., \& Prinz, W. (2001). The theory of event coding (TEC): A framework for perception and action planning. Behavioral and Brain Sciences, 24, 849-878. http://dx.doi .org/10.1017/S0140525X01000103

Hopson, J. W. (2003). General learning models: Timing without a clock. In W. H. Meck (Ed.), Functional and neural mechanisms of interval timing (pp. 23-60). Boca Raton, FL: CRC Press. http://dx.doi.org/10.1201/ 9780203009574.ch2

Houde, J. F., \& Jordan, M. I. (1998, February 20). Sensorimotor adaptation in speech production. Science, 279, 1213-1216. http://dx.doi.org/ 10.1126/science.279.5354.1213

Ivry, R. B., \& Richardson, T. C. (2002). Temporal control and coordination: The multiple timer model. Brain and Cognition, 48, 117-132. http://dx.doi.org/10.1006/brcg.2001.1308

Jones, J. A., \& Munhall, K. G. (2000). Perceptual calibration of F0 production: Evidence from feedback perturbation. Journal of the Acoustical Society of America, 108, 1246-1251. http://dx.doi.org/10.1121/1 .1288414

Jones, M. R., \& Boltz, M. (1989). Dynamic attending and responses to time. Psychological Review, 96, 459-491. http://dx.doi.org/10.1037/ 0033-295X.96.3.459

Jordan, M. I., \& Rumelhart, D. E. (1992). Forward models: Supervised learning with a distal teacher. Cognitive Science, 16, 307-354. http://dx .doi.org/10.1207/s15516709 $\operatorname{cog} 1603$

Kawai, K., Harada, K., Kato, K., Ueno, K., \& Sakuma, T. (2013). Experiment on adjustment of piano performance to room acoustics: Analysis of performance coded into MIDI data. Retrieved from http://www.caaaca.ca/conferences/isra2013/proceedings/Papers/P125.pdf

Kawato, M. (1999). Internal models for motor control and trajectory planning. Current Opinion in Neurobiology, 9, 718-727. http://dx.doi .org/10.1016/S0959-4388(99)00028-8
Kelso, J. A. S. (1997). Dynamic patterns: The self-organization of brain and behavior. Cambridge, MA: MIT Press.

Keough, D., Hawco, C., \& Jones, J. A. (2013). Auditory-motor adaptation to frequency-altered auditory feedback occurs when participants ignore feedback. BMC Neuroscience, 14, 25. http://dx.doi.org/10.1186/14712202-14-25

Kleiner, M., Brainard, D., \& Pelli, D. (2007). What's new in Psychtoolbox-3? [Abstract]. Perception, 36(Suppl.), 14.

Kolers, P. A., \& Brewster, J. M. (1985). Rhythms and responses. Journal of Experimental Psychology: Human Perception and Performance, 11 150-167. http://dx.doi.org/10.1037/0096-1523.11.2.150

Krakauer, J. W., \& Mazzoni, P. (2011). Human sensorimotor learning: Adaptation, skill, and beyond. Current Opinion in Neurobiology, 21, 636-644. http://dx.doi.org/10.1016/j.conb.2011.06.012

Krampe, R. T., Doumas, M., Lavrysen, A., \& Rapp, M. (2010). The costs of taking it slowly: Fast and slow movement timing in older age. Psychology and Aging, 25, 980-990. http://dx.doi.org/10.1037/ a0020090

Laird, N. M., \& Ware, J. H. (1982). Random-effects models for longitudinal data. Biometrics, 38, 963-974. http://dx.doi.org/10.2307/2529876

Lakoff, G., \& Johnson, M. (1999). Philosophy in the flesh: The embodied mind and its challenge to Western thought. New York: Basic Books.

Lalazar, H., \& Vaadia, E. (2008). Neural basis of sensorimotor learning: Modifying internal models. Current Opinion in Neurobiology, 18, 573 581. http://dx.doi.org/10.1016/j.conb.2008.11.003

LaRue, J. (2005). Initial learning of timing in combined serial movements and a no-movement situation. Music Perception, 22, 509-530. http://dx doi.org/10.1525/mp.2005.22.3.509

Lauber, B., \& Keller, M. (2014). Improving motor performance: Selected aspects of augmented feedback in exercise and health. European Journal of Sport Science, 14, 36-43. http://dx.doi.org/10.1080/17461391.2012 .725104

Lavie, N. (2010). Attention, distraction, and cognitive control under load. Current Directions in Psychological Science, 19, 143-148. http://dx.doi org/10.1177/0963721410370295

Lavie, N., Hirst, A., de Fockert, J. W., \& Viding, E. (2004). Load theory of selective attention and cognitive control. Journal of Experimental Psychology: General, 133, 339-354. http://dx.doi.org/10.1037/00963445.133.3.339

Leman, M. (2007). Embodied music cognition and mediation technology. Cambridge, MA: MIT Press.

Lewis, P. A., \& Miall, R. C. (2003). Distinct systems for automatic and cognitively controlled time measurement: Evidence from neuroimaging Current Opinion in Neurobiology, 13, 250-255. http://dx.doi.org/ 10.1016/S0959-4388(03)00036-9

Loehr, J. D., Large, E. W., \& Palmer, C. (2011). Temporal coordination and adaptation to rate change in music performance. Journal of Experimental Psychology: Human Perception and Performance, 37, 12921309. http://dx.doi.org/10.1037/a0023102

Maes, P.-J., Leman, M., Palmer, C., \& Wanderley, M. M. (2014). Actionbased effects on music perception. Frontiers in Psychology, 4, 1008 http://dx.doi.org/10.3389/fpsyg.2013.01008

Maes, P.-J., Wanderley, M. M., \& Palmer, C. (2015). The role of working memory in the temporal control of discrete and continuous movements. Experimental Brain Research, 233, 263-273. http://dx.doi.org/10.1007/ s00221-014-4108-5

Manning, F. C., \& Schutz, M. (2015). Movement enhances perceived timing in the absence of auditory feedback. Timing \& Time Perception, $3,1-10$

Mauk, M. D., \& Buonomano, D. V. (2004). The neural basis of temporal processing. Annual Review of Neuroscience, 27, 307-340. http://dx.doi org/10.1146/annurev.neuro.27.070203.144247 
Mollaei, F., Shiller, D. M., \& Gracco, V. L. (2013). Sensorimotor adaptation of speech in Parkinson's disease. Movement Disorders, 28, $1668-$ 1674. http://dx.doi.org/10.1002/mds.25588

Moravec, H. (1988). Mind children: The future of robot and human intelligence. Cambridge, MA: Harvard University Press.

Nijs, L., \& Leman, M. (2014). Interactive technologies in the instrumental music classroom: A longitudinal study with the music paint machine. Computers \& Education, 73, 40-59. http://dx.doi.org/10.1016/j .compedu.2013.11.008

Ogden, R. S., Salominaite, E., Jones, L. A., Fisk, J. E., \& Montgomery, C. (2011). The role of executive functions in human prospective interval timing. Acta Psychologica, 137, 352-358. http://dx.doi.org/10.1016/j .actpsy.2011.04.004

Palmer, C., Lidji, P., \& Peretz, I. (2014). Losing the beat: Deficits in temporal coordination. Philosophical Transactions of the Royal Society B: Biological Sciences, 369, 20130405. http://dx.doi.org/10.1098/rstb .2013 .0405

Palmer, C., \& Pfordresher, P. Q. (2003). Incremental planning in sequence production. Psychological Review, 110, 683-712.

Pfordresher, P. Q. (2006). Coordination of perception and action in music performance. Advances in Cognitive Psychology, 2, 183-198. http://dx .doi.org/10.2478/v10053-008-0054-8

Pfordresher, P. Q., \& Palmer, C. (2006). Effects of hearing the past, present, or future during music performance. Perception \& Psychophysics, 68, 362-376. http://dx.doi.org/10.3758/BF03193683

Pinheiro, J., \& Bates, D. (2000). Mixed-effects models in S and S-plus. New York: Springer. http://dx.doi.org/10.1007/978-1-4419-0318-1

Rattat, A.-C. (2010). Bidirectional interference between timing and concurrent memory processing in children. Journal of Experimental Child Psychology, 106, 145-162. http://dx.doi.org/10.1016/j.jecp.2010.02.001

Raveh, D., \& Lavie, N. (2015). Load-induced inattentional deafness. Attention, Perception, \& Psychophysics, 77, 483-492. http://dx.doi.org/ 10.3758/s13414-014-0776-2

Repp, B. H. (1999). Effects of auditory feedback deprivation on expressive piano performance. Music Perception, 16, 409-438. http://dx.doi.org/ $10.2307 / 40285802$

Repp, B. H., \& Steinman, S. R. (2010). Simultaneous event-based and emergent timing: Synchronization, continuation, and phase correction. Journal of Motor Behavior, 42, 111-126. http://dx.doi.org/10.1080/ 00222890903566418

Repp, B. H., \& Su, Y. H. (2013). Sensorimotor synchronization: A review of recent research (2006-2012). Psychonomic Bulletin \& Review, 20, 403-452. http://dx.doi.org/10.3758/s13423-012-0371-2

Robertson, S. D., Zelaznik, H. N., Lantero, D. A., Bojczyk, K. G., Spencer, R. M., Doffin, J. G., \& Schneidt, T. (1999). Correlations for timing consistency among tapping and drawing tasks: Evidence against a single timing process for motor control. Journal of Experimental Psychology: Human Perception and Performance, 25, 1316-1330. http://dx.doi.org/ 10.1037/0096-1523.25.5.1316

Roche, R., Wilms-Floet, A. M., Clark, J. E., \& Whitall, J. (2011). Auditory and visual information do not affect self-paced bilateral finger tapping in children with DCD. Human Movement Science, 30, 658-671. http://dx .doi.org/10.1016/j.humov.2010.11.008

Rodger, M. W. M., \& Craig, C. M. (2011). Timing movements to interval durations specified by discrete or continuous sounds. Experimental Brain Research, 214, 393-402. http://dx.doi.org/10.1007/s00221-0112837-2

Roerdink, M., Ridderikhoff, A., Peper, C. E., \& Beek, P. J. (2013). Informational and neuromuscular contributions to anchoring in rhythmic wrist cycling. Annals of Biomedical Engineering, 41, 1726-1739. http:// dx.doi.org/10.1007/s10439-012-0680-7

Rosati, G., Rodà, A., Avanzini, F., \& Masiero, S. (2013). On the role of auditory feedback in robot-assisted movement training after stroke:
Review of the literature. Computational Intelligence and Neuroscience, 2013, 586138. http://dx.doi.org/10.1155/2013/586138

Ross, J. M., \& Balasubramaniam, R. (2014). Physical and neural entrainment to rhythm: Human sensorimotor coordination across tasks and effector systems. Frontiers in Human Neuroscience, 8, 576. http://dx .doi.org/10.3389/fnhum.2014.00576

Shadmehr, R., Smith, M. A., \& Krakauer, J. W. (2010). Error correction, sensory prediction, and adaptation in motor control. Annual Review of Neuroscience, 33, 89-108. http://dx.doi.org/10.1146/annurev-neuro060909-153135

Shiller, D. M., \& Rochon, M. L. (2014). Auditory-perceptual learning improves speech motor adaptation in children. Journal of Experimental Psychology: Human Perception and Performance, 40, 1308-1315. http://dx.doi.org/10.1037/a0036660

Stenneken, P., Prinz, W., Cole, J., Paillard, J., \& Aschersleben, G. (2006) The effect of sensory feedback on the timing of movements: Evidence from deafferented patients. Brain Research, 1084, 123-131. http://dx .doi.org/10.1016/j.brainres.2006.02.057

Stevens, L. T. (1886). On the time-sense. Mind, 11, 393-404. http://dx.dol .org/10.1093/mind/os-XI.43.393

Studenka, B. E. (2015). Response to period shifts in tapping and circle drawing: A window into event and emergent components of continuous movement. Psychological Research, 79, 500-512. http://dx.doi.org/ 10.1007/s00426-014-0578-0

Studenka, B. E., Zelaznik, H. N., \& Balasubramaniam, R. (2012). The distinction between tapping and circle drawing with and without tactile feedback: An examination of the sources of timing variance. Quarterly Journal of Experimental Psychology, 65, 1086-1100. http://dx.doi.org/ 10.1080/17470218.2011.640404

Takahashi, N., \& Tsuzaki, M. (2008). Comparison of highly trained and less-trained pianists concerning utilization of auditory feedback. Acoustical Science and Technology, 29, 266-273. http://dx.doi.org/10.1250/ ast.29.266

Thelen, E. (1991). Timing in motor development as emergent process and product. In J. Fagard \& P. H. Wolff (Eds.), Advances in psychology (Vol 81, pp. 201-211). New York: Elsevier.

Torre, K., \& Balasubramaniam, R. (2009). Two different processes for sensorimotor synchronization in continuous and discontinuous rhythmic movements. Experimental Brain Research, 199, 157-166. http://dx.doi .org/10.1007/s00221-009-1991-2

Turvey, M. T. (1977). Preliminaries to a theory of action with reference to vision. In L. Shaw \& J. Bransford (Eds.), Perceiving, acting, and knowing: Toward an ecological psychology (pp. 211-265). Hillsdale, NJ: Erlbaum.

van der Steen, M. C., \& Keller, P. E. (2013). The ADaptation and Anticipation Model (ADAM) of sensorimotor synchronization. Frontiers in Human Neuroscience, 7, 253. http://dx.doi.org/10.3389/fnhum .2013 .00253

van Vugt, F. T., \& Tillmann, B. (2015). Auditory feedback in error-based learning of motor regularity. Brain Research, 1606, 54-67. http://dx.doi .org/10.1016/j.brainres.2015.02.026

Varela, F. J., Rosch, E., \& Thompson, E. (1991). The embodied mind: Cognitive science and human experience. Cambridge, MA: MIT Press.

Varlet, M., Marin, L., Issartel, J., Schmidt, R. C., \& Bardy, B. G. (2012) Continuity of visual and auditory rhythms influences sensorimotor coordination. PLOS ONE, 7(9), e44082. http://dx.doi.org/10.1371/journal .pone.0044082

Verbeke, G., \& Molenberghs, G. (2000). Linear mixed models for longitudinal data. New York: Springer-Verlag.

Villacorta, V. M., Perkell, J. S., \& Guenther, F. H. (2007). Sensorimotor adaptation to feedback perturbations of vowel acoustics and its relation to perception. Journal of the Acoustical Society of America, 122, $2306-$ 2319. http://dx.doi.org/10.1121/1.2773966 
Warren, W. H. (2006). The dynamics of perception and action. Psychological Review, 113, 358-389. http://dx.doi.org/10.1037/0033-295X .113 .2 .358

Welch, R. B. (1986). Adaptation of space perception. In K. R. Boff, L. Kaufman, \& J. P. Thomas (Eds.), Handbook of perception and human performance, Vol. 1. Sensory processes and perception (pp. 24-45). New York: Wiley.

Wing, A. M., \& Kristofferson, A. B. (1973). Response delays and the timing of discrete motor responses. Perception \& Psychophysics, 14, 5-12. http://dx.doi.org/10.3758/BF03198607

Wolpert, D. M., Ghahramani, Z., \& Jordan, M. I. (1995, September 29). An internal model for sensorimotor integration. Science, 269, 1880-1882. http://dx.doi.org/10.1126/science.7569931
Zelaznik, H. N., Spencer, R. M. C., \& Ivry, R. B. (2002). Dissociation of explicit and implicit timing in repetitive tapping and drawing movements. Journal of Experimental Psychology: Human Perception and Performance, 28, 575-588. http://dx.doi.org/10.1037/0096-1523.28.3 .575

Zelaznik, H. N., Spencer, R. M. C., \& Ivry, R. B. (2008). Behavioral analysis of human movement timing. In S. Grondin (Ed.), Psychology of time (pp. 233-260). Bingley, England: Emerald.

Received November 11, 2014

Revision received May 8, 2015

Accepted May 8, 2015 